\title{
Methodology for Obtaining Linear State Space Building Energy Simulation Models
}

\author{
Damien Picard $^{1} \quad$ Filip Jorissen $^{1,2} \quad$ Lieve Helsen $^{1,2}$ \\ ${ }^{1}$ Mechanical engineering, KU Leuven, Belgium, \{damien.picard, filip.jorissen\}@kuleuven.be \\ ${ }^{2}$ EnergyVille, Waterschei, Belgium, lieve.helsen@energyville.be
}

\begin{abstract}
Optimal climate control for building systems is facilitated by linear, low-order models of the building structure and of its Heating, Ventilation and Air Conditioning (HVAC) systems. However, obtaining these models in a practical form is often difficult, which greatly hampers the commercial implementation of model predictive controllers. This work describes a methodology for obtaining a linear State Space Model (SSM) of Building Energy Simulation (BES) models, consisting of walls, windows, floors and the zone air. The methodology uses the Modelica library IDEAS to develop a BES model, including its non-linearities, and automates its linearisation. The Dymola function linearize2 is used to generate the state space formulation, facilitating further mathematical manipulations, or simulation in different environments. Optionally this model can then be reduced for control purposes using model order reduction (MOR) techniques. The methodology is illustrated for the zone air temperature in an office building. For this case, the absolute error between the non-linear BES and its SSM remains under $1 \mathrm{~K}$ and its yearly average is $0.21 \mathrm{~K}$. The original 50 states SSM could furthermore be reduced to 16 states without significant loss of accuracy.

Keywords: model predictive control, Dymola, building energy simulation, linearisation, model order reduction.
\end{abstract}

\section{Introduction}

Building climate control uses around $18 \%$ of the total end energy in Europe (Perez-Lombard et al., 2008). One way of reducing energy use is to develop more efficient control algorithms for the production and distribution of heat and cold in buildings. Recent research has shown that (near) optimal controllers such as Model Predictive Control (MPC) can greatly improve the energy efficiency of buildings compared to traditional rulebased-controllers (Gyalistras and Gwerder, 2009; Verhelst, 2012). However, its practical implementation is hampered due to the difficulty of finding a controller model that is simple enough to allow optimization within a reasonable computation time but still accurate enough to correctly predict the building behaviour. Linear models are preferred since efficient optimization algorithms can then be used (Kummert, 2001; Sturzenegger et al., 2012).

Controller models are often obtained using system identification, i.e. fitting reduced order models based on measurement data. Obtaining controller models for buildings is an active research topic due to the complexity of the systems and due to the difficulty or even impossibility of performing experiments allowing the identification of multi-input, multi-output building models (Sturzenegger et al., 2014). An alternative approach is to create models based on physical insight and knowledge about the system. Lehmann et al. (2013) showed that building energy simulation (BES) models are only weakly non-linear. They set up a relative complex linear model based uniquely on physical data, which was able to mimic the non-linear TRNSYS BES model with an error smaller than $1 \mathrm{~K}$. The accuracy of the model is not enough for design purposes but it is sufficient for MPC or sensitivity analysis. Sturzenegger et al. (2014) automated their approach for deriving state space models for MPC applications using the BRCM Matlab toolbox. The toolbox needs a considerable amount of information such as an EnergyPlus input file.

In this work, we propose an automated way of obtaining accurate linear BES models based on a non-linear model implementation in Dymola using the IDEAS library (Baetens et al., 2015). Section 2 describes the nonlinearities of BES models together with common simplifications and Section 3 explains the linearisation technique. Section 4 describes the linearisation methodology in IDEAS and Section 5 shows a validation of the methodology. Section 6 briefly discusses a model order reduction technique for the linear model and their use for optimal controllers. Main conclusions are summarized in Section 7. 


\section{Non-linearities in Building Energy Simulation Models and Common Simplifications}

Typically, BES models contain three major sources of non-linearities. The first is longwave radiation, which is typically described using the Stefan-Boltzmann law. The second is the absorption of incident solar radiation by windows, which is a function of the incidence angle. The third is convective heat transfer, which is usually described using correlations for the convective heat transfer coefficient. These non-linear equations are first described in this section, then a linearisation technique is proposed. Other non-linearities in real buildings exist (e.g temperature dependent emissivity, pressure dependent air leakage, ...) but they are rarely modelled. They will not be treated in this work.

Radiation Radiation is described by the non-linear Stefan-Boltzmann law which is given by Eq. 1 for two grey-bodies with surface areas $A_{1}$ and $A_{2}$.

$$
\dot{Q}_{1 \rightarrow 2}(t)=\sigma F_{1 \rightarrow 2} A_{1}\left(T_{1}^{4}(t)-T_{2}^{4}(t)\right)
$$

$\dot{Q}_{1 \rightarrow 2}$ and $F_{1 \rightarrow 2}$ are the heat transferred from surface 1 to 2 and their view factor respectively, $\sigma=5.670373 \times$ $10^{-8} \mathrm{~W} /\left(\mathrm{m}^{2} \cdot \mathrm{K}^{4}\right)$ the Stefan-Boltzmann constant, and $T_{i}$ the temperature of body $i$.

Radiative heat transfer between room surfaces is often approximated using the Mean Radiant Temperature model (e.g. in TRNSYS TYPE 56 ( S.A. Klein et al., 2010)) or using the Radiant Star Temperature model (e.g. in IDEAS (Baetens et al., 2015)) since it greatly simplifies the computations without a significant loss in accuracy (Liesen and Pedersen, 1997). This radiant star temperature $T_{\text {star }}$ is derived from the energy conservation equation in the radiant node and the temperature of each surface $A_{k}$ is calculated using a distribution coefficient $R_{k}$ :

$$
\dot{Q}_{k \rightarrow \text { star }}(t)=\frac{\sigma A_{k}}{R_{k}}\left(T_{k}^{4}(t)-T_{\mathrm{star}}^{4}(t)\right)
$$

Eq. 2 is often linearised around nominal temperatures $T_{k, n o m}$ and $T_{\text {star,nom }}$ (Eq. 3 ), which is an accurate approximation for small temperature differences. Figure 1 shows the approximation error for the heat exchange between two black bodies with view factor equal to one.

$$
\begin{array}{r}
\dot{Q}_{k \rightarrow \operatorname{star}}(t) \simeq c\left(T_{k}(t)-T_{\mathrm{star}}(t)\right) \\
c=\frac{\sigma A_{k}}{R_{k}}\left(\left(T_{k, \text { nom }}+T_{\text {star }, \text { nom }}\right)\left(T_{k, \text { nom }}^{2}+T_{\text {star }, \text { nom }}^{2}\right)\right.
\end{array}
$$

The longwave radiation heat flow $\dot{Q}_{l w, k}(t)$ between exterior surface $k$ of the building with longwave emissivity

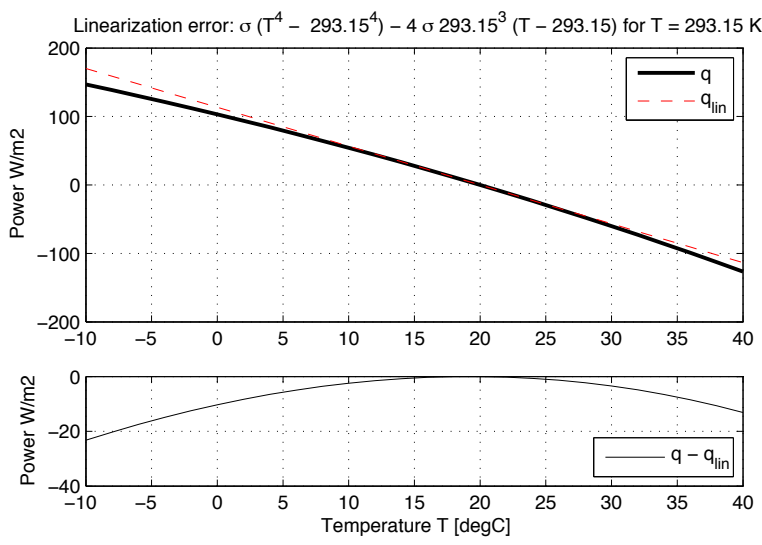

Figure 1. Error made by the linearisation of the radiative heat transfer equation between two black bodies with view factor one.

$\varepsilon_{l w, k}$ and its environment can be modelled as:

$$
\begin{aligned}
\dot{Q}_{l w, k}(t)= & \sigma \varepsilon_{l w, k} A_{k}\left(T_{s, k}^{4}(t)-F_{c e, k} T_{c e}^{4}(t)\right. \\
& \left.-\left(1-F_{c e, k}\right) T_{d b}^{4}(t)\right) \\
F_{c e, k} & =\frac{1+\cos i_{k}}{2}
\end{aligned}
$$

with $T_{s, k}(t), T_{c e}(t), T_{d b}(t)$ the surface, celestial dome and dry bulb temperature respectively, $F_{c e, k}$ the view factor between the surface $k$ and the celestial dome, and $i_{k}$ the inclination of the surface. This equation is linearised by default in IDEAS as:

$$
\begin{aligned}
\dot{Q}_{l w, k}(t) & \simeq c\left(T_{s, k}(t)-\right. \\
& \left.\sqrt[4]{F_{c e, k} T_{c e}^{4}(t)+\left(1-F_{c e, k}\right) T_{d b}^{4}(t)}\right)
\end{aligned}
$$

with $c$ a parameter defined similar to Eq. 4 .

Finally, the shortwave solar irradiation absorbed by exterior surface $k$ equals:

$$
\dot{Q}_{s w, k}(t)=\varepsilon_{s w, k} A_{k} E_{e, k}(t)
$$

with $E_{e, k}(t)$ the incident solar irradiation on surface $A_{k}$ as a function of time.

Absorption and transmission through glazing Heat absorbed or transferred through windows is typically highly non-linear as it depends on the spectral properties of the window, on the angle of incidence of the sun and on possible shading. Typically, the window properties are pre-computed using specialized software and delivered as an input to the simulation software. IDEAS uses the software Window 4.0 (Finlayson et al., 1993) to pre-compute window spectral properties but it computes the amount of absorbed and transmitted light during the simulation, requiring trigonometrical transformations and lookup tables, which are non-linear functions. 
Convective heat transfer Two types of convective heat transfer are present in buildings: exterior, forced convection by the wind, and interior, natural convection when forced ventilation is absent.

In IDEAS, the external convective heat transfer rate $\dot{Q}_{c v}(t)$ between an exterior surface with area $A_{k}$ and the outdoor air is based on Defraeye et al. (2011):

$$
\begin{aligned}
\dot{Q}_{c v, k}(t) & =h_{c v}(t) A_{k}\left(T_{d b}(t)-T_{s, k}(t)\right) \\
h_{c v}(t) & =\max \left\{5.01\left(v_{10}(t)\right)^{0.85}, 5.6\right\} \mathrm{W} / \mathrm{m}^{2} \mathrm{~K}
\end{aligned}
$$

with convective heat transfer coefficient $h_{c v}(t)$, dry bulb ambient temperature $T_{d b}(t)$, surface temperature $T_{s, k}(t)$ and the undisturbed wind speed at 10 meters above the ground $v_{10}(t)$.

Eq. 8 is non-linear even if the convection coefficient is an input due to the multiplication of input with input $\left(h_{c v}(t) T_{d b}(t)\right)$ and inputs with state $\left(h_{c v}(t) T_{s, k}(t)\right)$. If the nominal values of $T_{d b}(t)$ and $T_{s, k}(t)$ are equal, Eq. 8 can be linearised as:

$$
\dot{Q}_{c v, k}(t) \simeq \bar{h}_{c v} A_{k}\left(T_{d b}(t)-T_{s, k}(t)\right)
$$

with $\bar{h}_{c v}$ the yearly average of the exterior convection coefficient.

The interior convective heat transfer rate of a wall, ceiling or floor with surface area $A_{k}$ and an air node is computed as:

$$
\begin{aligned}
\dot{Q}_{c v, k}(t) & =h_{c v, k}(t) A_{k}\left(T_{d b}(t)-T_{s, k}(t)\right) \\
h_{c v, k}(t) & =n_{1, k} D_{k}^{n_{2, k}}\left|T_{d b}(t)-T_{s, k}(t)\right|^{n_{3, k}}
\end{aligned}
$$

with $D_{k}$ the hydraulic diameter, and coefficients $n_{i, k}$. The value of the coefficients are $n_{1: 3}=$ $\{1.823,-0.121,0.293\}$ for vertical surfaces, $n_{1: 3}=$ $\{2.175,-0.076,0.308\}$ for heated floors and cooled ceilings and $n_{1: 3}=\{0.704,-0.601,0.133\}$ for cooled floors and heated ceilings (Awbi and Hatton, 1999).

These interior convection equations can be linearised in IDEAS using an average value for $h_{c v}$ :

$$
h_{c v, k} \simeq n_{1, k} D_{k}^{n_{2, k}}\left|\Delta T_{\text {nom }}\right|^{n_{3, k}}
$$

with $\Delta T_{\text {nom }}$ the nominal temperature difference.

Heat diffusion through walls and floors Heat transfer through walls and floors is characterized by convective and radiative heat transfer at the surfaces and conduction through the solid layers. The latter is governed by a partial differential equation (PDE). It extends in three spatial dimensions and in time. However, the heat transfer through walls and floor can often be approximated using a one dimensional PDE due to the low thickness to height and width ratio. The equations can then either be solved using discrete Laplace transform (e.g. TRNSYS) or using a finite volume method (e.g. EnergyPlus (Strand et al., 1999)). In IDEAS, the finite volume method is used, leading to a set of linear equations.

\section{Linearisation Technique}

The linearisation of a function consists of the first order term of the Taylor expansion of this function around a working point. Given a deterministic non-linear dynamic system:

$$
\begin{aligned}
& \dot{x}=f(x, u) \\
& y=g(x, u)
\end{aligned}
$$

where $x \in \mathbb{R}^{n_{x}}$ are the states, $\dot{x}$ are their derivatives, $u \in$ $\mathbb{R}^{n_{u}}$ the inputs, and $y \in \mathbb{R}^{n_{y}}$ the outputs. The linearisation of Eq. 12 around point $p_{\star} \triangleq\left(x_{\star}, u_{\star}\right)$ is defined as:

$$
\begin{aligned}
\dot{x} & =f\left(p_{\star}\right)+\left.\frac{\partial f}{\partial x}\right|_{p_{\star}}\left(x-x_{\star}\right)+\left.\frac{\partial f}{\partial u}\right|_{p_{\star}}\left(u-u_{\star}\right) \\
& \triangleq f\left(p_{\star}\right)+\mathbf{A} \tilde{x}+\mathbf{B} \tilde{u} \\
y & =g\left(p_{\star}\right)+\left.\frac{\partial g}{\partial x}\right|_{p_{\star}}\left(x-x_{\star}\right)+\left.\frac{\partial g}{\partial u}\right|_{p_{\star}}\left(u-u_{\star}\right) \\
& \triangleq g\left(p_{\star}\right)+\mathbf{C} \tilde{x}+\mathbf{D} \tilde{u}
\end{aligned}
$$

where $\mathbf{A}, \mathbf{B}, \mathbf{C}, \mathbf{D}$ are constant matrices.

The Dymola built-in function linearize2 of the Modelica Linear System 2 library provides the possibility of linearising Modelica models (Otter, 2014). The hybrid differential-algebraic equation system is treated as an ordinary differential equation system at the linearisation point and the partial derivatives of the functions $f$ and $g$ are obtained by evaluation of the analytical Jacobian if it is available. Otherwise a central difference method is used. The function can also be used to transform a linear model into a SSM.

It should be noted that even for a linear system, the linearisation point $p_{\star}$ used by the function linearize 2 should be chosen carefully to avoid numerical noise. The states $x_{\star}$ can be set using initial equations or start values. The inputs $u_{\star}$ can be set using start values. The default start value for the inputs in Dymola is zero which can lead to significant error when evaluating the derivatives using the central difference method.

\section{Linearisation Methodology in IDEAS}

This section describes how IDEAS was adapted to automatically obtain a state space formulation of a BES model in Dymola. Firstly the linearization of the equations is discussed, followed by the model structure requirements for SSM's. Finally the SSM structure is described.

\subsection{Linearisation of the equations}

Here we describe how the non-linear equations of the Modelica BES models are conditionally linearised or 


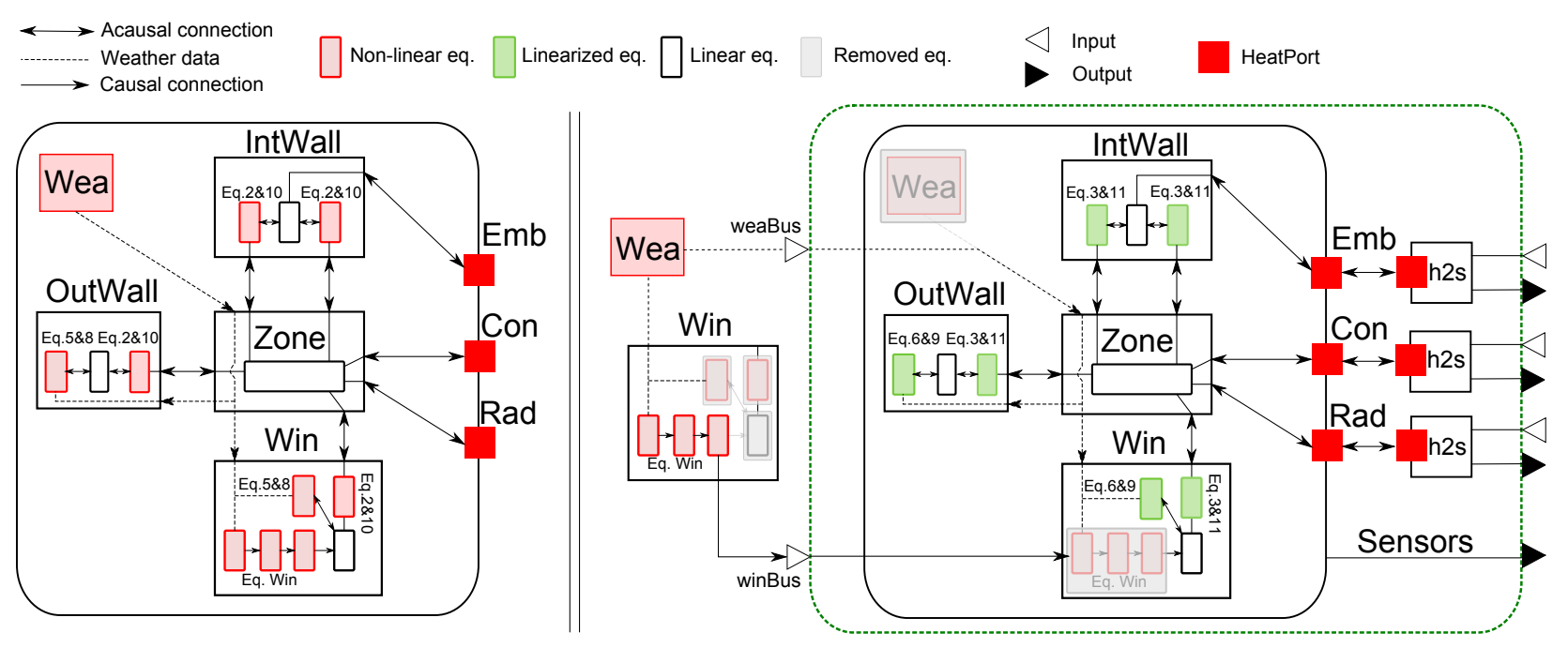

Figure 2. Left: original model with non-linear equations. Right: Adjusted model structure with moved and/or linearized nonlinear equations. Component models are outer wall 'OutWall', interior wall 'IntWall', Window 'Win', weather model inputs 'Wea' and HeatPorts embedded (Emb), convective (Con) and radiative (Rad). White triangles represent inputs to the model, whereas black triangles represent outputs of the model.

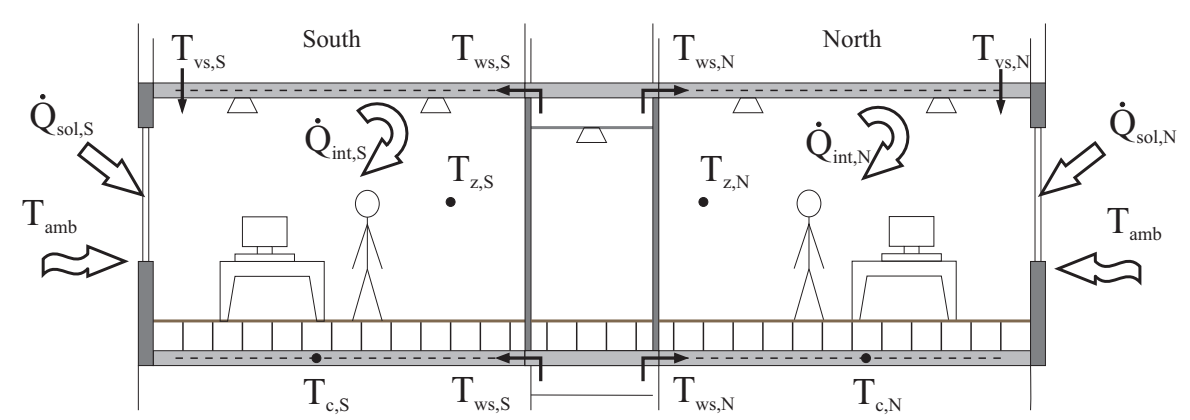

Figure 3. Illustration of the office building section, (Sourbron et al. (2013), p 5)

moved outside the model and replaced by model inputs. Note that the moved equations should not depend on any state variables.

Radiation As described in the previous section, all longwave radiation equations can be linearised accurately. If linearise $=$ true, Eq. 2 and Eq. 5 are replaced by Eq. 3 and Eq. 6, respectively, where the square root term is transformed into a model input for each different orientations and inclination. The solar irradiation $E_{e}^{(k)}(t)$ required for the shortwave absorption is also converted into a model input per orientation and inclination.

Window models Window models contain equations for calculating the solar irradiance, the impact of shading and the amount of heat that is absorbed and transmitted through the window. These are non-linear equations indicated in Figure 2 by 'Eq. Win'. Linearising these equations would introduce large errors. Linearising them at may for instance have the consequence that the solar position and corresponding incidence angles become fixed, which can cause a large underestimation of the so- lar gains for windows. Therefore the absorbed and transmitted heat flow rates are calculated outside of the model and they are inputs to the linearised model, as indicated in the right of Figure 2. Each window model is instantiated twice, once inside and once outside of the linearised model. The grey boxes in Figure 2 indicate which equations are removed and replaced by inputs. Note that the window model is thereby split into two parts. A bus connector winBus for each of the $n_{\text {win }}$ windows is used for connecting the inputs.

Convective heat transfer The interior convective heat transfer is linearised using Eq. 11. $\Delta T_{\text {nom }}$ was chosen equal to the mean absolute temperature difference between the window or wall and the zone air temperature. The exterior convective heat transfer coefficient is simplified by using the yearly average convective heat transfer coefficient $\bar{h}_{c v}$. These linearisations are indicated on Figure 2 using green rectangles.

The remaining model equations, like thermal conduction equations, are already linear and they are retained. 


\subsection{State space formulation}

In the previous paragraphs all non-linear equations are removed from the building envelope model. This linear model needs to be converted into state space format. This requires that all exterior connections are either inputs or outputs, otherwise Dymola does not detect the connections. However, HeatPort connections Emb, Con and Rad contain variables $T$ and Q_flow that do not specify whether they are inputs or outputs. Each HeatPort for the room thermal gains is therefore connected to an input-output block $\mathrm{h} 2 \mathrm{~s}$, which either sets heat flow rate Q_flow to a fixed input and temperature $T$ to an output or the other way around.

In order to propagate weather data inputs to all submodels, one weather bus weaBus with prefix input is connected to each zone. The zone further propagates this data to all its connected surfaces (walls, windows, ...) as indicated by the dotted lines in Figure 2.

\subsection{State space model structure}

All non-linear equations are now removed and all connections are either defined as an input or as an output. The state space formulation can now be obtained by using the linearize2 function on the model containing all components of the dashed green box in Figure 2. This function returns matrices $\mathbf{A}, \mathbf{B}, \mathbf{C}$ and $\mathbf{D}$. The SSM inputs $u$ are the heat flow rate or temperature for thermal gains of the zones, the weather bus and $n_{\text {win }}$ window buses. Outputs are either the temperature or the heat flow rate of the transformed HeatPorts. Additional outputs can be defined in the linearised model by adding Realoutput components.

\section{Validation}

In this section, the methodology is applied to a test case. The case is firstly described after which the methodology is validated.

Case description The validation uses the cut-out of a typical office building with South and North oriented facades described by Sourbron et al. (2013) (See Figure 3). We only consider the building structure, which consists of three zones (a corridor, a south-oriented and a north-oriented zone) each equipped with a thermally activated ceiling and floor composed of multiple layers (floor tiles, air layer, screed, and reinforced concrete), two external walls composed of multiple layers (plaster, concrete blocks, mineral wool, and bricks), and two windows. Each zone has a convective and a radiative heat gain input and heat can also be injected at the core of the thermally activated building parts.

The model is implemented with all details above in Modelica using the IDEAS library (Baetens et al., 2015).
Table 1. Comparison between three models based on equation types and equation formats.

\begin{tabular}{lrrr}
\hline & Ref & Lin & SSM \\
\hline Convection & non-linear & linear & linear \\
Radiation & non-linear & linear & linear \\
Model inputs & non-linear & non-linear & non-linear \\
Other equations & linear & linear & linear \\
SSM formulation & no & no & yes \\
\hline
\end{tabular}

The model has 8434 variables and 50 differentiated states. Once linearised, the model has 52 inputs. The model uses the weather data of Uccle (Belgium).

Each of the heat flow rate inputs is set equal to the sum of the two sinusoids of Equations 14-15, with $t=0$ at new year. The sinusoid with a period of one day and one year respectively represent internal gains, and heating or cooling delivered by the HVAC system. The sinusoid parameters are tuned such that the zone temperature remains around $22{ }^{\circ} \mathrm{C}$.

$$
\begin{aligned}
& \sin _{1}=4+4 \sin \left(\frac{2 \pi t}{86400}-\frac{\pi}{2}\right) \\
& \sin _{2}=13 \sin \left(\frac{2 \pi t}{31536000}-1.4\right)
\end{aligned}
$$

Model description In order to validate the methodology, the zone temperatures of three models are compared. The reference model is the IDEAS model with non-linear radiative heat transfer (Eq. 3 and 6), temperature-dependent interior convection (Eq. 11) and wind speed dependent exterior convection (Eq. 8).

The second model is identical to the reference model but it uses the linearised equations for the radiation and interior and exterior convection. The model is then fully linear except for its inputs.

Note that the linearisation of the exterior convection coefficient can cause a heat flow rate error of more to 150 $\mathrm{W} / \mathrm{m}^{2}$ due to the wide range of $h_{c v}$ (from 7 to $55 \mathrm{~W} / \mathrm{m}^{2} \mathrm{~K}$ ) and the potentially large difference between the ambient dry bulb temperature and the surface temperature. For the given example, the maximum deviation is $141 \mathrm{~W} / \mathrm{m}^{2}$. This error culminates when both wind speed and solar radiation are high, which causes both a high heat transfer rate and a high surface temperature.

The third model is the state space version of the second model. The SSM is loaded into Dymola using Modelica.Blocks. Continuous. Statespace.

Note that the difference between the third and the second model should be around the solver tolerance.

A comparison between the equation types and formats of the three models is given in Table 1. 

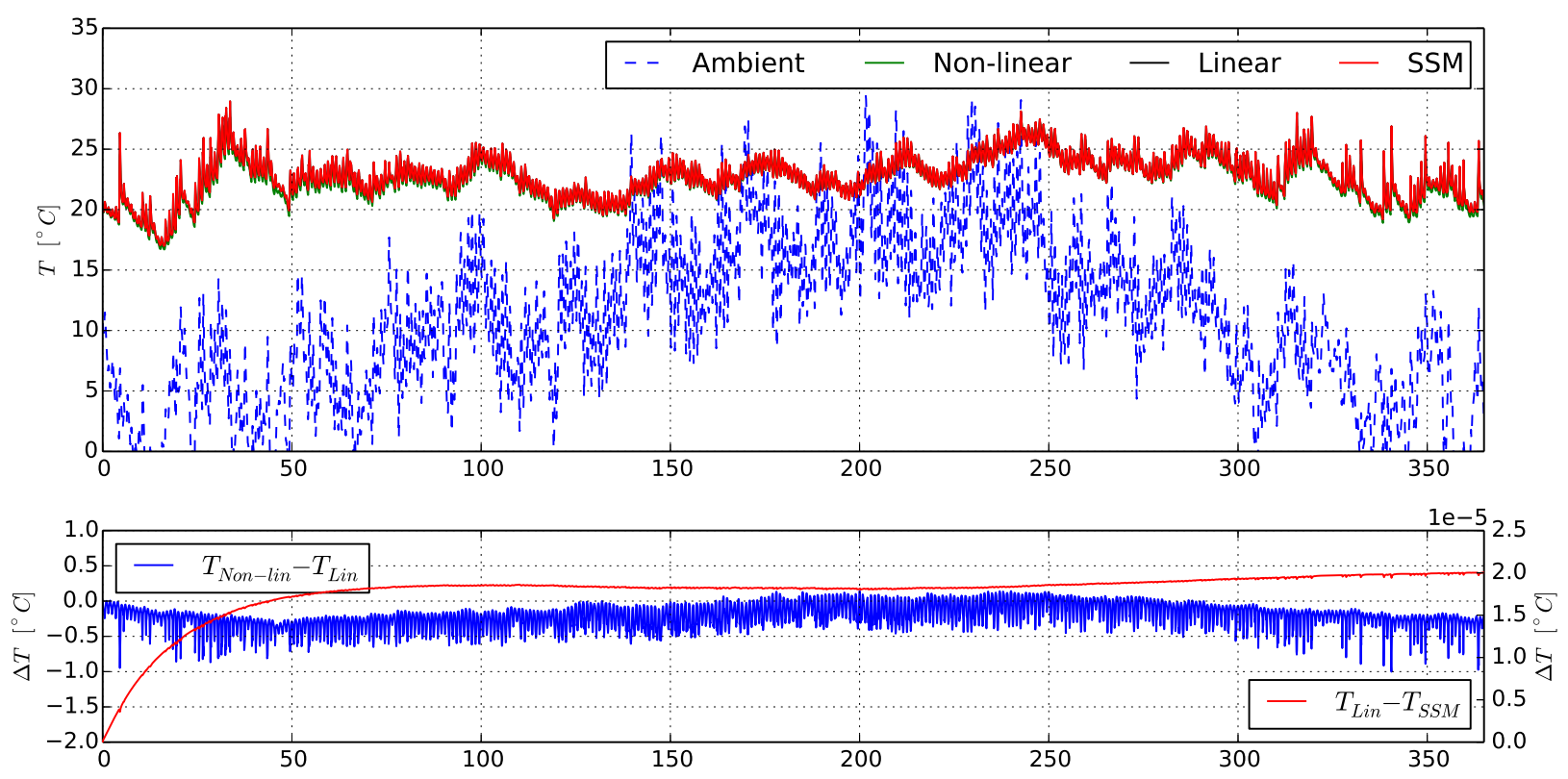

Figure 4. Result comparison between three model types for a one year simulation. The top graph shows absolute temperatures, while the other two graphs show absolute temperature differences.

Model comparison The three model versions are simulated in one model for a whole year using solver Dassl with a tolerance of $10^{-6}$. The zone air temperatures are then compared. Figure 4 shows the southern zone temperature of the different models, the average error of the three zone air temperatures of the reference model and the linear model, and this average error for the linear model and its SSM. The figure shows that the zone temperature is excited over a realistic range. The CPU time is also compared ${ }^{1}$. Normalized CPU times $t_{\text {norm }}$ are computed by subtracting the 'CPUtime' required for a simulation that only computes the building model inputs. The CPU time ratio $r_{i}$ is computed based on the non-linear reference case: $r_{i}=\frac{t_{\text {norm,ref }}}{t_{\text {norm, }}}$. The total computation time for the reference case is $290 \mathrm{~s}$.

Figure 4 shows that the linear model is a good approximation of the non-linear model as the absolute error remains smaller than $1 \mathrm{~K}$ and its average is close to zero. This justifies the often made linear approximations in building modelling. Figure 4 also shows that the transformation of the linear model into a SSM does not introduce significant errors, as expected. This indicates that the model equations were successfully extracted by the linearize2 function.

The linear model is faster than the non-linear model with $r_{\text {lin }}=1.8$. This can be expected because linear equations typically require less operations and do not require non-linear algebraic loops to be solved. Interestingly, the SSM is much faster with a $r_{S S M}=8.5$. This is

\footnotetext{
${ }^{1}$ Simulations are performed using Dymola 2016 and Euler integration using a fixed time step of $10 \mathrm{~s}$ and a duration of $10^{7}$ seconds. Euler integration is chosen to ensure that the same number of time steps is performed.
}

because the state space model contains only 50 states and therefore only 50 equations. The linear model contains 50 states and 453 additional $^{2}$ algebraic variables, which also need to be computed, often requiring the analytical solution of linear systems of equations.

These results suggest that the symbolic processing could be improved, resulting in faster models.

\section{Model Order Reduction}

The obtained SSM of Section 5 is accurate but a large number of states is used. This might be problematic for model-based optimal controllers such as MPC. In this section, we apply a MOR technique for different orders and we investigate their simulation accuracy compared to the original model. The comparison is extended by implementing a state observer for each ROM and by computing the 48-hours ahead prediction performance. The prediction performance is an indicator for the efficiency of the MPC which uses the model predictions to optimize the inputs of the system.

The different ROM's are obtained by applying the Matlab function reduce to the SSM, using the default balance algorithm (balancmr). The simulation performance is compared using the mininum, maximum, mean and nominal root mean square error (NRMSE) (Eq. 16) between the original SSM and the ROM's for each of the three zones. The errors are calculated over a period of 100 days. The applied heat inputs and the gains are computed as a sum of sinusoids with 30 frequencies and

\footnotetext{
${ }^{2}$ The translated linear model contains 453 'time-varying variables' more than the translated SSM model.
} 
realistic amplitudes. The weather-related inputs are computed using a typical year of Uccle (Belgium).

$$
\operatorname{NRMSE}^{(n)}=100\left(1-\frac{\left\|y-\hat{y}^{(n)}\right\|}{\|y-\bar{y}\|}\right)
$$

with $y$ the output signal, $\bar{y}$ its time averaged value, and $\hat{y}^{(n)}$ the output signal of the $n$ order ROM.

Figure 5 shows the comparison for the reduced order models of different orders. For this particular example, the error rapidly decreases with the model order and it becomes negligible for ROM's with $n \geq 15$. The error on the south zone, which is irradiated by more direct sun light than the north zone, is the highest. We therefore conclude that the MOR technique can for this case successfully decrease the number of states without significant loss of accuracy but that a minimal number of states is necessary to correctly capture the faster dynamics of the system. These dynamics correspond to small thermal capacities of the different surfaces excited by the sun. This result was expected as the MOR technique typically removes the small eigenvalues of the system, responsible for the fast dynamics.

Note that by applying model reduction, the size of the SSM matrices decreases but the original matrices sparsity is lost. It is therefore not interesting to use reduced order model in Dymola as the number of additions and multiplication increase thereby. However, the loss of sparsity for optimal controller model is not an issue, since the required conversion from the continuous to the discrete time domain already removes that sparsity of the matrices.

\section{Conclusion}

This paper presents an approach for deriving linear state space models from BES models using the IDEAS library and Dymola. To this end, weakly non-linear equations are linearised. The remaining non-linear equations can be evaluated outside of the model since they do not depend on the model states. The resulting model is linearised using the Dymola function linearize2, which derives the state space matrices. The errors made by linearising the models are found to be acceptable. The SSM can be reduced using model order reduction techniques. For the tested case, the order of the model could be reduced by a factor three without significant loss of prediction accuracy. An important advantage of the presented methodology is that it automates the conversion of IDEAS BES models into state space formulation which can then be used for different purposes or by different programs.

The current implementation still presents some drawbacks that can be solved in the future. So far, the model can only have four different perpendicular orientations and all surfaces should either be horizontal or vertical.
Furthermore, the Medium in the zone should be simple air without any species concentration. Finally ventilation can only be modelled using heat flow inputs and not using mass/energy transport equations.

\section{Acknowledgments}

The authors acknowledge the financial support by the Agency for Innovation by Science and Technology in Flanders (IWT) for the PhD work of F. Jorissen (contract number 131012). The authors acknowledge the financial support by IWT and WTCB in the frame of the IWT-VIS Traject SMART GEOTHERM focusing on integration of thermal energy storage and thermal inertia in geothermal concepts for smart heating and cooling of (medium) large buildings. This work emerged from the Annex 60 project, an international project conducted under the umbrella of the International Energy Agency (IEA) within the Energy in Buildings and Communities (EBC) Programme.

\section{References}

S.A. Klein et al. TRNSYS 17, A Transient System Simulation Program. Solar Energy Laboratory, University of Wisconsin, Madison, USA, http://sel.me.wisc.edu/trnsys, 2010.

H.B. Awbi and a. Hatton. Natural convection from heated room surfaces. Energy and Buildings, 30(3):233-244, August 1999.

R. Baetens, R. De Coninck, F. Jorissen, D. Picard, L. Helsen, and D. Saelens. OpenIDEAS - An open framework for integrated district energy simulations. In Submitted to Building simulation 2015, Hyderabad, 2015.

T. Defraeye, B. Blocken, and J. Carmeliet. Convective heat transfer coefficients for exterior building surfaces: Existing correlations and CFD modelling. Energy Conversion and Management, 52(1):512 - 522, 2011.

E. U. Finlayson, D. K. Arasteh, C. Huizenga, M. D. Rubin, and M. S. Reilly. Window 4.0: Documentation of calculation procedures. Technical report, 1993.

D. Gyalistras and M. Gwerder. Use of Weather and Occupancy Forecasts for optimal building climate control, two years progress report. Technical report, 2009.

M. Kummert. Contribution to the application of modern control techniques to solar buildings. Simulation-based approach and experimental validation. $\mathrm{PhD}$ thesis, Fondation Universitaire Luxembourgeoise, 2001.

B. Lehmann, D. Gyalistras, M. Gwerder, K. Wirth, and S. Carl. Intermediate complexity model for Model Predictive Control of Integrated Room Automation. Energy and Buildings, 58(0):250 - 262, 2013. 

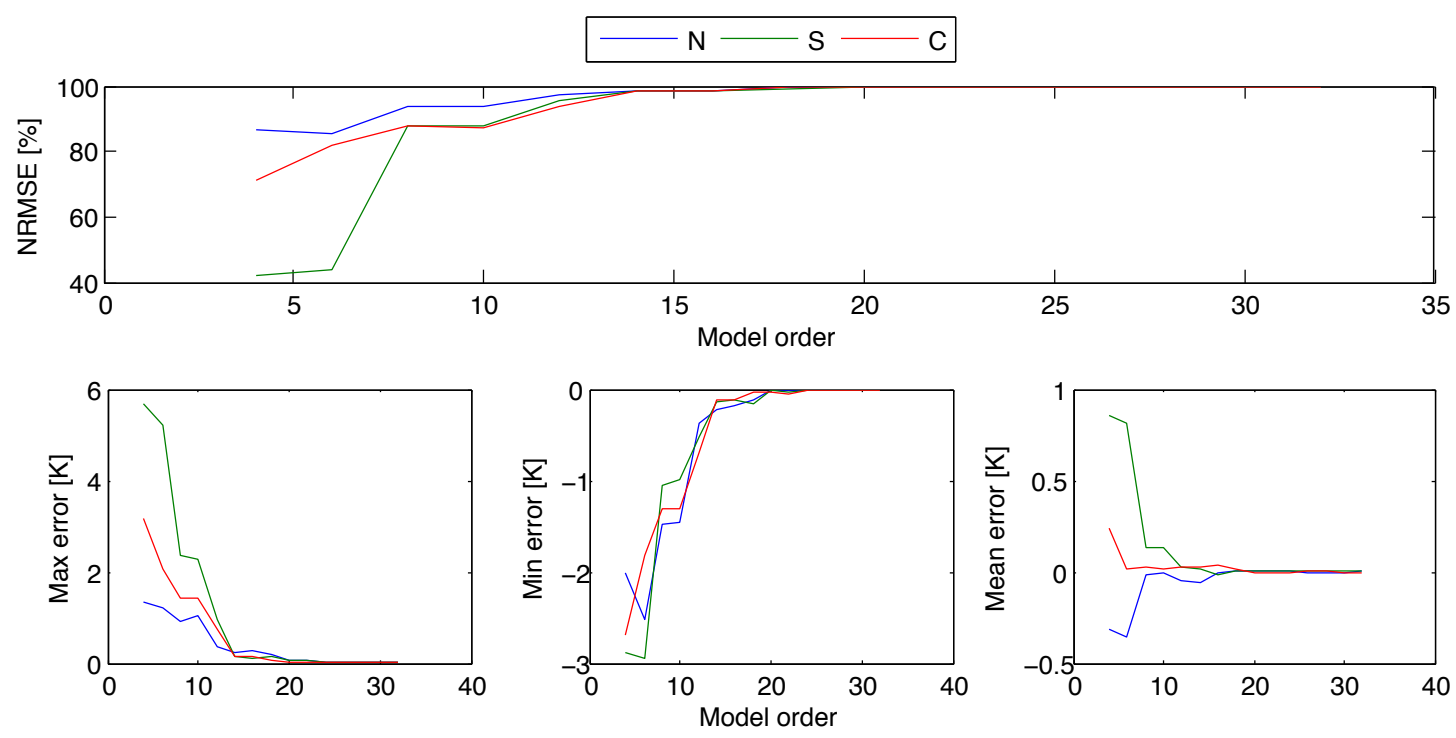

Figure 5. Nominal root mean square error (NRMSE), maximum, minimum and mean error between the original state space model of 50 states and the reduced order model of different order for the north $(\mathrm{N})$, south $(\mathrm{S})$ and corridor $(\mathrm{C})$ zones.

R.J. Liesen and C.O. Pedersen. An evaluation of inside surface heat balance models for cooling load calculations. Technical report, American Society of Heating, Refrigerating and AirConditioning Engineers, Inc., Atlanta, GA (United States), 1997.

M. Otter. Modelica_linearsystems2, 2014. URL https://github.com/modelica/Modelica_ Linearsystems 2 .git.

L. Perez-Lombard, J. Ortiz, and C. Pout. A review on buildings energy consumption information. Energy and Buildings, 40 (3):394-398, 2008.

M. Sourbron, C. Verhelst, and L. Helsen. Building models for model predictive control of office buildings with concrete core activation. Journal of Building Performance Simulation, 6(3):175-198, 2013.

R. Strand, F. Winkelmann, F. Buhl, J. Huang, R. Liesen, C. Pedersen, D. Fisher, R. Taylor, D. Crawley, and L. Lawrie. Enhancing and Extending the Capabilities of the Building Heat Balance Simulation Technique for use in EnergyPlus. In in Proceedings of Building Simulation'99, Volume II, pages 653-660, Kyoto, Japan, 1999.

D. Sturzenegger, D. Gyalistras, M. Morari, and Smith R. Semiautomated modular modeling of buildings for model predictive control. In BuildSys 2012 - Workshop of ACM SenSys Conference, Toronto, Canada, 2012.

D. Sturzenegger, D. Gyalistras, V. Semeraro, M. Morari, and R. Smith. BRCM Matlab Toolbox: Model Generation for Model Predictive Building Control. In American Control Conference, pages 1063-1069, Portland, June 2014.

C. Verhelst. Model Predictive Control of Ground Coupled Heat Pump Systems for Office Buildings. PhD thesis, KU Leuven, Belgium, 2012. 